\title{
Travelling wave phenomena in non-linear diffusion degenerate Nagumo equations
}

\author{
Faustino Sánchez-Garduño ${ }^{2}$, Philip K. Maini ${ }^{1}$ \\ ${ }^{1}$ Centre for Mathematical Biology, Mathematical Institute, University of Oxford, \\ 24-29 St. Giles', Oxford OX1 3LB, UK \\ ${ }^{2}$ Departamento de Matemáticas, Facultad de Ciencias, UNAM, Circuito Exterior, \\ C.U., México 04510, D.F., Mexico
}

Received 15 December 1995; received in revised form 14 May 1996

\begin{abstract}
In this paper we study the existence of one-dimensional travelling wave solutions $u(x, t)=\phi(x-c t)$ for the non-linear degenerate (at $u=0)$ reaction-diffusion equation $u_{t}=\left[D(u) u_{x}\right]_{x}+g(u)$ where $g$ is a generalisation of the Nagumo equation arising in nerve conduction theory, as well as describing the Allee effect. We use a dynamical systems approach to prove: 1. the global bifurcation of a heteroclinic cycle (two monotone stationary front solutions), for $c=0,2$. The existence of a unique value $c^{*}>0$ of $c$ for which $\phi\left(x-c^{*} t\right)$ is a travelling wave solution of sharp type and 3. A continuum of monotone and oscillatory fronts for $c \neq c^{*}$. We present some numerical simulations of the phase portrait in travelling wave coordinates and on the full partial differential equation.
\end{abstract}

Key words: Sharp fronts - Degenerate diffusion - Hamiltonian - Bifurcation of heteroclinic trajectories

\section{Introduction}

In this paper we deal with the problem of looking for travelling wave solutions (t.w.s.) $u(x, t)=\phi(x-c t) \equiv \phi(\xi)$ for the equation

$$
\frac{\partial u}{\partial t}=\frac{\partial}{\partial x}\left[D(u) \frac{\partial u}{\partial x}\right]+g(u), \quad \forall(x, t) \in \boldsymbol{R} \times \boldsymbol{R}^{+},
$$

where for a given real number $\alpha \in(0,1)$, the functions $D$ and $g$ defined on the interval $[0,1]$ satisfy the conditions:

1. $g(0)=g(\alpha)=g(1)=0, g(u)<0 \forall u \in(0, \alpha), g(u)>0 \forall u \in(\alpha, 1)$,

2. $g \in C_{[0,1]}^{2}, g^{\prime}(0)<0, g^{\prime}(\alpha)>0, g^{\prime}(1)<0$ and $g^{\prime \prime}(0)>0$,

3. $D(0)=0, D(u)>0 \forall u \in(0,1]$,

4. $D \in C_{[0,1]}^{2}, D^{\prime}(u)>0 \forall u \in[0,1]$ and $D^{\prime \prime}(0)>0$.

We also require the conditions: $u(x, 0)=u_{0}(x)$ with $0 \leqq u_{0}(x) \leqq 1$ where $u_{0}$ is a piecewise differentiable function on $R$ and $0 \leqq \phi(\xi) \leqq 1 \forall \xi \in(-\infty,+\infty)$. 
Note that because of condition 3, equation (1) is of parabolic type for all $u>0$ and degenerates into an ODE at $u=0$. The degeneracy of equation (1) results in two specific features of its solutions which do not appear in equations with constant diffusion coefficient: 1 . the finite speed of propagation through space, and 2. the existence of weak solutions, particularly those solutions with discontinuous space derivative.

Note that $u_{1}(x, t) \equiv 0, u_{2}(x, t) \equiv \alpha$ and $u_{3}(x, t) \equiv 1$ are homogeneous and stationary solutions of equation (1). They play a key role in the asymptotic behaviour of the t.w.s. of this equation.

Given the appropriate physical interpretation of $u$ in equation (1) this can be seen as a generalization of those equations arising as models for different biological systems. Thus (1) could represent the space-time dynamics of a population (with population density $u$ ) showing an Allee effect and whose individuals disperse to avoid crowded areas (see [3, 4, 6, 9, 17]). Meanwhile, if we interpret $u$ as the probability of occurrence of the allele $A$ in a population with two alleles ( $A$ and $a$ ), equation (1) is a generalization of that describing the dispersion of the gene $A$ in the heterozygote inferior case (see $[2,18]$ ). Finally, if we interpret $u$ as a membrane potential in a nerve axon, equation (1) is a generalization of Nagumo's equation arising in nerve conduction models (see [16]).

Although very detailed analysis of travelling wave solutions (t.w.s.) to the constant diffusion equation with non-linear kinetic term having the geometric properties 1-2 above already exists in the literature, this is not the case for generalized degenerate non-linear Nagumo reaction-diffusion equations.

Since the classic papers $[10,15]$ on t.w.s. for a constant diffusion equation with quadratic-like kinetic part, much research has been developed to try to extend this analysis to more general reaction-diffusion equations. Particular cases of a density-dependent diffusion coefficient vanishing at $u=0$, with reactive part having the qualitative properties listed above, have also been studied. For example, several authors have analysed the equation $u_{t}=\left[u^{m}\right]_{x x}+u(1-u)(u-\alpha)$, where $m>1$ and $\alpha \in(0,1)$, for specific values of $m$ (see $[3,11,13,14]$ ). Their results focus on the existence of a critical value, $c^{*}>0$, of $c$ for which the above equation has a sharp type solution and on the convergence to t.w.s.

In this paper we extend previous analysis on existence of different types of t.w.s. to the general equation (1). We use a dynamical systems theory approach, in which the problem of finding t.w.s. for (1) satisfying suitable boundary conditions, is equivalent to determining the set of parameters (which includes the speed $c$ ) for which there exist heteroclinic (or homoclinic) trajectories for an autonomous system of two ordinary differential equations (ODEs). This system arises from transforming the original problem into travelling wave coordinates.

The paper is organized as follows: Sect. 2 contains a result on the sign of the speed $c$ of the t.w.s. of equation (1). There we also state the main result of this paper. In Sect. 3 we outline the non-linear local analysis to obtain the local phase portrait of the transformed system of ODEs for the case $c \geqq 0$. In 
Sect. 4 we carry out the global analysis for the case $c=0$. We exhibit there the bifurcation of a heteroclinic cycle and illustrate our results with an example. In Sect. 5 we begin the analysis of the whole phase portrait of the ODE system for $c>0$ by obtaining some results on global behaviour which are related to the existence of some t.w.s. of front (oscillatory and monotone) type. In Sect. 6 we use a couple of previous results obtained by the authors (see [20]) to prove the existence and uniqueness of a critical value, $c^{*}$, of $c$ for which there exists a travelling wave solution of sharp type. We illustrate the analytical results of the paper by considering an example.

Since the proofs of some results of this paper use similar techniques developed in a previous publication ([20]), we will be concerned here more with the consequences of the theory, rather than with developing the methodological aspects. In this sense the present work can be seen as an extension of the aforementioned reference. Full details can be found in [19].

\section{Statement of the main result}

Our analysis starts by calculating the sign of the speed of t.w.s. of (1). For this let $\mathscr{D}:[0,1] \rightarrow \boldsymbol{R}$ be the function defined as:

where $w=\phi(\xi)$.

$$
\mathscr{D}(\phi)=\int_{0}^{\phi} D(w) g(w) d w,
$$

Proposition 2.1. Suppose sufficient conditions of smoothness of the functions $D$ and $g$ on the interval $[0,1]$. Assume that equation (1) has t.w.s. whose behaviour is as sketched in Fig. 1. For Figs. 1(a-c), $c>0$. For Fig. 1(d), $c>0$ if and only if $\mathscr{D}(1)>0$.

Proof. This can be obtained following a similar methodology to that given in [20]. We omit the details.

Before we state the main result of this paper, we must mention that we have adopted here the definition of sharp type solution of equation (1) from [20], which corresponds to a weak solution type for equation (1).

Our theorem is:

Theorem 1. If the functions $D$ and $g$ satisfy conditions 1-4 given in Sect. 1, then there exists a unique value, $c^{*}>0$, of the speed $c$, such that equation (1):

1. has for $c=0$ : (a) an isolated pulse based at $P_{0}$ if $\mathscr{D}(1)>0$; (b) an isolated pulse based at $P_{1}$ if $\mathscr{D}(1)<0$; (c) two stationary monotonic fronts: one connecting the states 0 and 1 and the other connecting 1 to 0 if $\mathscr{D}(1)=0$,

2. has an oscillatory front from 0 to $\alpha$ and another from 1 to $\alpha$ for each $c$ such that $0<c<c^{*}<\sqrt{4 D(\alpha) g^{\prime}(\alpha)}$,

3. has a unique travelling wave solution of sharp type from 1 to 0 for a critical value, $c^{*}$, of the speed $c$. For this value of $c$ there exists an oscillatory travelling wave from 0 to $\alpha$, 

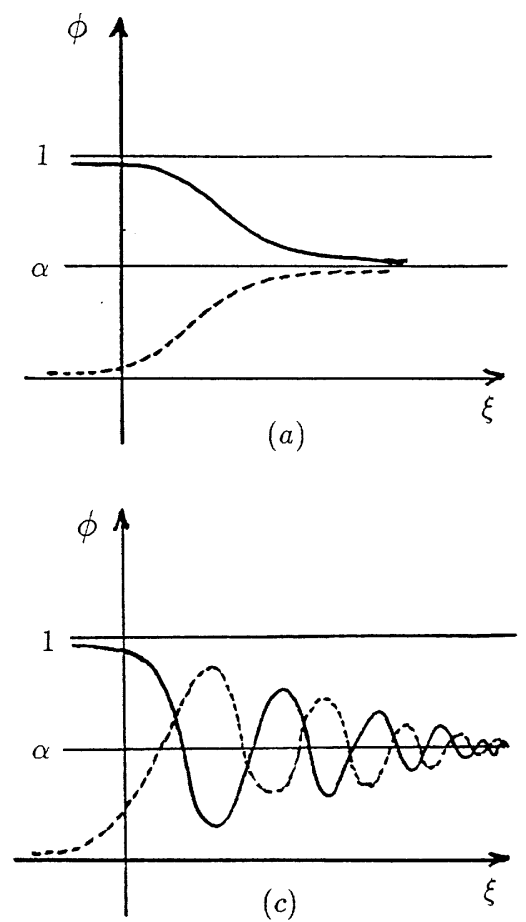
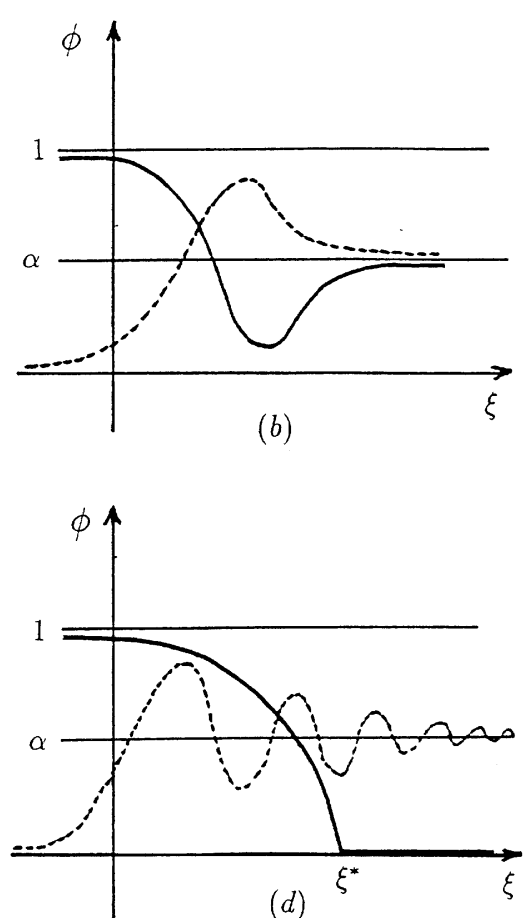

(d)

Fig. 1a-d. Different behaviours of t.w.s. for equation (1) depicted by continuous and broken lines. For the waves depicted in $\mathbf{a}-\mathbf{c}$ the speed $c$ is greater than zero. For $\mathbf{d}, c>0$ if and only if $\mathscr{D}(1)>0$. The solution shown here is the so-called sharp type travelling wave (see $[20]$ for the definition)

4. does not possess t.w.s. connecting the homogeneous and stationary steady states: i) $u(x, t) \equiv 1$ and $u(x, t) \equiv 0$, for $\mathscr{D}(1) \leqq 0$ and $c>0$ and ii) $u(x, t) \equiv 0$ and $u(x, t) \equiv 1$ for $\mathscr{D}(1)>0$,

5. has two oscillatory travelling fronts for $c^{*}<c<\sqrt{4 D(\alpha) g^{\prime}(\alpha)}$ : one from 0 to $\alpha$ and another from 1 to $\alpha$,

6. has a monotonic decreasing front from 1 to $\alpha$ for each $c$ such that

$$
c \geqq \sqrt{4 D(\alpha) g^{\prime}(\alpha)} .
$$

For the same values of $c$ it has a monotonic increasing front from 0 to $\alpha$.

In the following sections we outline the proof of Theorem 1 and refer the reader to [19] for fuller details.

\section{Local phase portrait for $c \geqq 0$}

We follow the aforementioned classical t.w.s. analysis introduced in [15]. The ODE system can be obtained as follows. If a travelling wave solution 
$u(x, t)=\phi(x-c t)$ of $(1)$ exists, it must satisfy the ODE equation

$$
D(\phi(\xi)) \phi^{\prime \prime}(\xi)+c \phi^{\prime}(\xi)+D^{\prime}(\phi)\left[\phi^{\prime}(\xi)\right]^{2}+g(\phi(\xi))=0
$$

where the dash on $D$ means the derivative with respect to $\phi$, while that on $\phi$ means the derivative with respect to $\xi$. By setting $v=\phi^{\prime}$ equation (3) can be written as the singular (at $\phi=0$ ) ODE system

$$
\begin{aligned}
\phi^{\prime} & =v \\
D(\phi) v^{\prime} & =-c v-D^{\prime}(\phi) v^{2}-g(\phi) .
\end{aligned}
$$

The singularity can be removed by introducing (see $[3,20])$ the parameter $\tau=\tau(\xi)$ into (4) such that

$$
\frac{d \tau}{d \xi}=\frac{1}{D(\phi(\xi))} .
$$

If we define $\phi(\xi) \equiv \phi(\tau(\xi)), v(\tau) \equiv v(\tau(\xi))$ and denote by dot the derivative with respect to $\tau$, system (4) can be re-written as the following non-singular system

$$
\begin{aligned}
\dot{\phi} & =D(\phi) v \\
\dot{v} & =-c v-D^{\prime}(\phi) v^{2}-g(\phi) .
\end{aligned}
$$

Equation (5), together with the condition $0 \leqq \phi(\tau) \leqq 1 \forall \tau \in(-\infty,+\infty)$, constitutes the re-statement in travelling wave coordinates of the original problem.

We begin the phase portrait analysis by noting that system (5) has three equilibria: $P_{0}=(0,0), P_{1}=(1,0), P_{\alpha}=(\alpha, 0)$ and $P_{c}=\left(0,-c / D^{\prime}(0)\right)$. As a consequence of the existence of four equilibria, equations (5) have a greater richness of dynamics than those of the corresponding ODE system associated with the degenerate Fisher-KPP equation, which has only three (see [20]). Nevertheless, some of the techniques developed in that reference can be extended to the present case.

The local phase portrait of (5) can be obtained by using its local approximation around each equilibria. For $P_{1}, P_{\alpha}$ and $P_{c}$ the linear analysis is sufficient to determine the local behaviour. However, since $P_{0}$ is a nonhyperbolic point for all values of $c$, we need higher order terms in the approximation (see [1] and the application of the Centre Manifold Theorem (see [7]). The result of the linear and the nonlinear analyses is:

Case 1. If $c=0, P_{0}$ is a saddle-like point, $P_{\alpha}$ a centre and $P_{1}$ a saddle point,

Case 2. If $c>0, P_{0}$ is a saddle-node, $P_{c}$ and $P_{1}$ are saddle points, and $P_{\alpha}$ is a locally stable: i) node if $c^{2} \geqq 4 D(\alpha) g^{\prime}(\alpha)$ or ii) focus if $c^{2}<4 D(\alpha) g^{\prime}(\alpha)$.

The local phase portrait of (5) is shown in Fig. 2. 

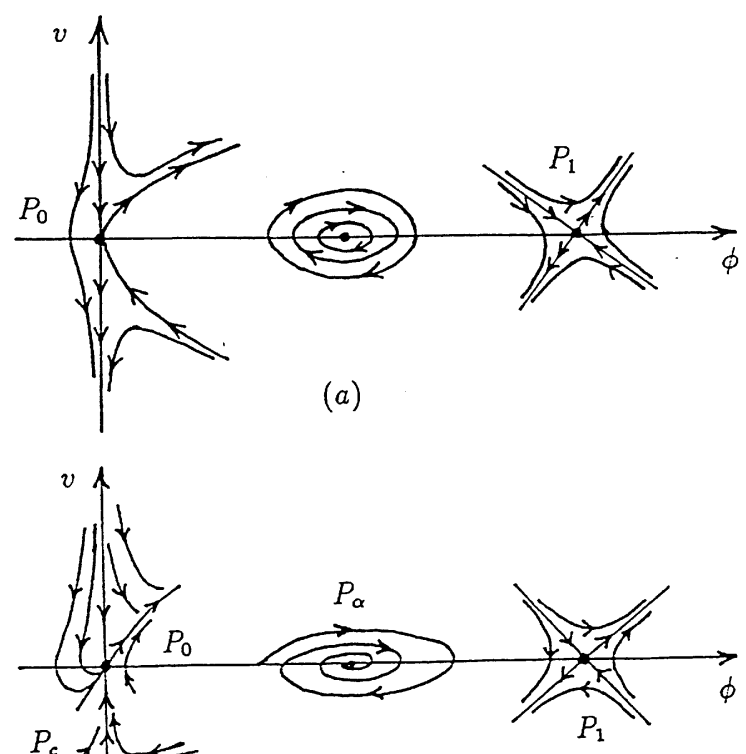

(b)
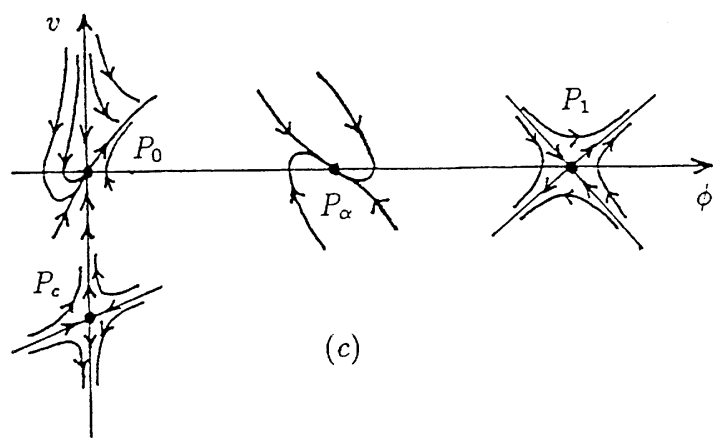

(c)

Fig. 2a-c. Local phase portrait of (5) for different values of $c$ : $\mathbf{a} c=0, \mathbf{b} c^{2}<4 D(\alpha) g^{\prime}(\alpha)$ and c $c^{2} \geqq 4 D(\alpha) g^{\prime}(\alpha)$

In the next sections we determine the global phase portrait of (5) for $c \geqq 0$ focusing on determining the existence of different heteroclinic and homoclinic trajectories. We begin with the case $c=0$.

\section{Bifurcation of a heteroclinic cycle for $c=0$}

We note that multiplying the right-hand side of (5) (with $c=0$ ), by $D$, the system can be transformed into a hamiltonian-like system whose trajectories coincide on the first and fourth quadrants of the $\phi v$-plane with those of (5). 
The hamiltonian of the modified system is

$$
H(\phi, v)=\frac{1}{2}[D(\phi) v]^{2}+\int_{0}^{\phi} D(w) g(w) d w .
$$

By using the geometrical properties of the level curves $H(0,0) \equiv 0$ and $H(1,0) \equiv \mathscr{D}(1)$ of $H$ (which in turn depend on those of $D$ and $g$ ), one can prove the following lemma which, actually, gives us the proof of item 1 in Theorem 1.

Lemma 4.1. System (5) with $c=0$ has a:

1. Homoclinic trajectory based in $P_{0}$, if $\mathscr{D}(1)>0$,

2. Heteroclinic cycle from $P_{0}$ to $P_{1}$ and from $P_{1}$ to $P_{0}$, if $\mathscr{D}(1)=0$,

3. Homoclinic trajectory based in $P_{1}$, if $\mathscr{D}(1)<0$.

The solutions of (1) associated with the trajectories mentioned in the above lemma are illustrated in Fig. 3.

In the following example we illustrate the results contained in Lemma 4.1.

Example 1. Here we consider the equation

$$
\frac{\partial u}{\partial t}=\frac{\partial}{\partial x}\left[\left(\beta u+u^{2}\right) \frac{\partial u}{\partial x}\right]+u(1-u)(u-\alpha),
$$

where $\beta>0$ and $\alpha \in(0,1)$.

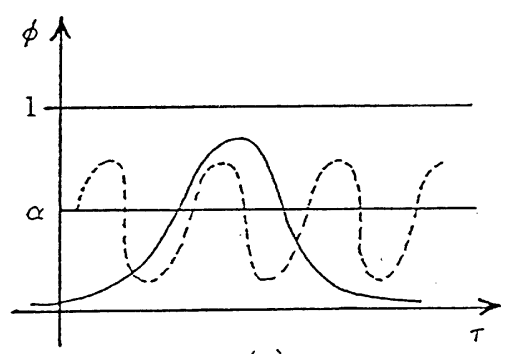

(a)

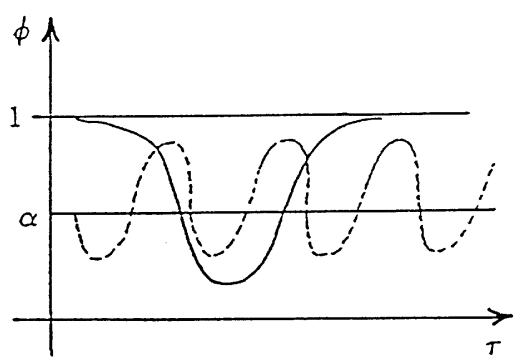

(c)

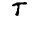

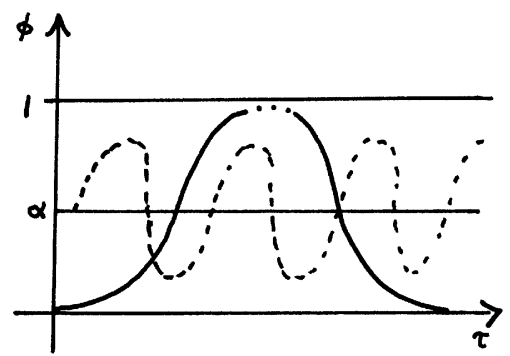

(b)
Fig. 3a-c. Sketch of the solutions

$u(x, t)=\phi(x-c t)$ of $(1)$ for $c=0$ corresponding to the different conditions mentioned in Lemma 4.1 for: a $\mathscr{D}(1)>0, \mathbf{b} \mathscr{D}(1)=0$ and $\mathbf{c} \mathscr{D}(1)<0$. The broken lines represent the periodic solutions associated with the closed trajectories of (5). In b the dots at the top of the monotonic fronts represent the asymptotic behaviour of both fronts as $\tau \rightarrow+\infty$ and $\tau \rightarrow-\infty$, respectively 
For $c=0$ system (5) takes the form

$$
\begin{aligned}
\dot{\phi} & =\left(\beta \phi+\phi^{2}\right) v \\
\dot{v} & =-(\beta+2 \phi) v^{2}-\phi(1-\phi)(\phi-\alpha),
\end{aligned}
$$

The condition $\mathscr{D}(1)=\int_{0}^{1} D(w) g(w) d w=0$ holds if and only if $\alpha$ and $\beta$ are related as follows

$$
\alpha(\beta)=\frac{3 \beta+2}{3+5 \beta} .
$$

This relationship divides the plane of parameters into regions for which system (8) has one homoclinic connection based at $P_{0}$ or based at $P_{1}$. The plane of the parameters and the phase portrait of system (8) showing the bifurcation of the heteroclinic cycle are illustrated in Fig. 4.
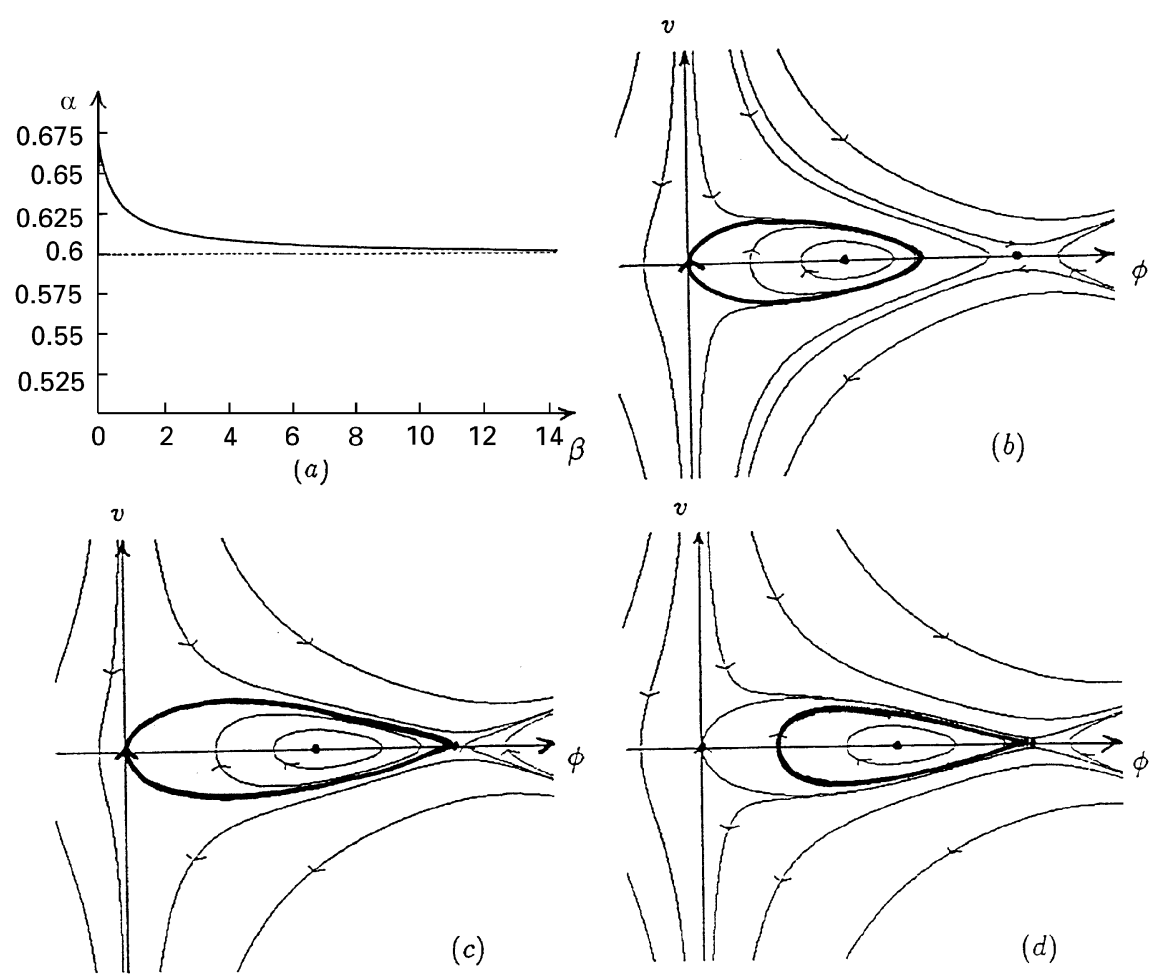

Fig. 4a-d. Dynamics of the system (8): a $\alpha-\beta$ parameter space. b The homoclinic trajectory based in $P_{0}$ for $\mathscr{D}(1)>0(0<\alpha<\alpha(\beta))$ (thick line). c Heteroclinic cycle from $P_{0}$ to $P_{1}$ (thick line) and from $P_{1}$ to $P_{0}$ for $\mathscr{D}(1)=0(\alpha=\alpha(\beta))$ (thick line). d Homoclinic trajectory based in $P_{1}$ for $\mathscr{D}(1)<0(\alpha>\alpha(\beta))$ (thick line). Numerically, it is difficult to pick the stable or unstable manifolds for this case, so we illustrate the trajectory by hand. 


\section{Monotonicity and the existence of some fronts}

In this section we prove some monotonicity properties of the paths of the trajectories of system (5) and the existence of certain types of heteroclinic connections. We introduce the following notation: Let $W_{c}^{u}\left(P_{1}\right)$ and $W_{c}^{s}\left(P_{c}\right)$ denote the left unstable and the right stable manifolds of (5) at $P_{1}$ and $P_{c}$, respectively.

We begin the global analysis by addressing the behaviour of the vertical null-clines of (5):

$$
V_{1}(\phi)=\frac{-c+\sqrt{c^{2}-4 D^{\prime}(\phi) g(\phi)}}{2 D^{\prime}(\phi)} \text { and } \quad V_{2}(\phi)=\frac{-c-\sqrt{c^{2}-4 D^{\prime}(\phi) g(\phi)}}{2 D^{\prime}(\phi)}
$$

Their shape changes with $c$ as is shown in Fig. 5 in which we have also drawn the vector field defined by (5), the way in which $W_{c}^{u}\left(P_{1}\right)$ is leaving $P_{1}$ and the form in which $W_{c}^{s}\left(P_{c}\right)$ approaches $P_{c}$.

Before we continue with the proof we first give a brief conceptual overview of the analysis presented in this and subsequent sections. This can be summarized in the following three cases corresponding to different values of $c$ :

1. For sufficiently small positive values of $c$, the phase portrait of (5) looks like that illustrated in Fig. 6(a),

2. For sufficiently large values of $c$, namely $c>0$ such that $c^{2} \geqq M \equiv \max [4 D(\phi) g(\phi)]$, where the maximum is taken on $[0,1]$, the phase portrait of (5) is as in Fig. 6(b),

3. For intermediate values of $c$ we have a rich dynamics, including the existence and uniqueness of a saddle-saddle heteroclinic trajectory for a critical value, $c^{*}$, of $c$.

Firstly we eliminate the parameter $\tau$ in (5). This leads to the equation

$$
\frac{d v}{d \phi}=\frac{-c v-D^{\prime}(\phi) v^{2}-g(\phi)}{D(\phi) v} .
$$

Hereafter $v_{1}$ and $v_{2}$ will denote two solutions of equation (11) corresponding to two positive values, $c_{1}$ and $c_{2}$, of the speed, $c$, respectively. Clearly, $v_{1}$ and $v_{2}$ satisfy $v_{1}(1)=v_{2}(1)=0$.

Denoting by ' the derivative of $v$ with respect to $\phi$, it is straightforward to verify that

$$
v_{2}^{\prime}-v_{1}^{\prime}=\frac{\left(c_{1}-c_{2}\right) v_{1} v_{2}+\left(v_{1}^{2} v_{2}-v_{2}^{2} v_{1}\right) D^{\prime}+\left(v_{2}-v_{1}\right) g}{D v_{1} v_{2}} .
$$

We now define the functions $\mathscr{G}_{1}$ and $\mathscr{G}_{0}$ such that

$$
\mathscr{G}_{1}(\phi)=\left[v_{2}(\phi)-v_{1}(\phi)\right] D(\phi) \exp \left[-\int_{\alpha}^{\phi} \frac{g(s)}{v_{1}(s) v_{2}(s) D(s)} d s\right],
$$



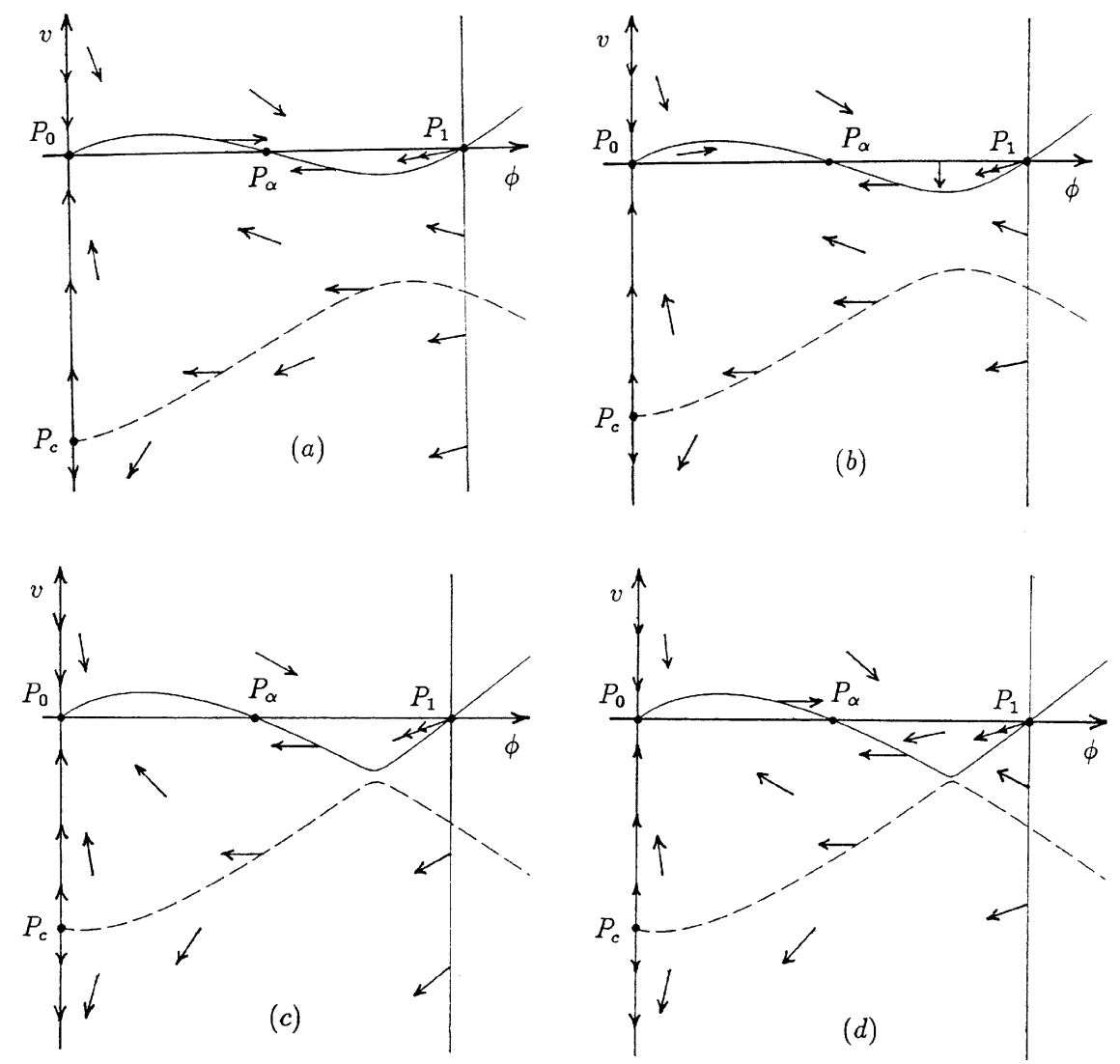

Fig. 5a-d. Qualitative behaviour of the vertical null-clines of (5) for different values of $c$ : a $c^{2}>M \equiv \max \left[4 D^{\prime}(\phi) g(\phi)\right]$. Here $V_{2}^{\prime}(0)>0, \mathbf{b} c^{2}=M$ and $\mathbf{c} c^{2}<M$. Continuous line represents $V_{1}$ and broken line represents $V_{2}$

and $\mathscr{G}_{0}(\phi)=-\mathscr{G}_{1}(\phi)$. By noting that $\mathscr{G}_{1}(\phi) \rightarrow 0$ as $\phi \rightarrow 1^{-}$and $\mathscr{G}_{0}(\phi) \rightarrow 0$ as $\phi \rightarrow 0^{+}$we can prove the following lemma on the monotonicity properties of the solutions of equation (11) as $c$ changes:

Lemma 5.1. Let $c_{1}$ and $c_{2}$ be two values of $c$ such that $c_{1}<c_{2}$. If $v_{1}$ and $v_{2}$ are both:

1. Negative on $[\alpha, 1)$ and satisfy $v_{1}(1)=v_{2}(1)=0$, then $v_{1}(\phi)<v_{2}(\phi)$ $\forall \phi \in(0,1)$,

2. Positive on $(0, \alpha]$ and satisfy $v_{1}(0)=v_{2}(0)=0$, then $v_{1}(\phi)>$ $v_{2}(\phi) \forall \phi \in(0,1)$,

3. Negative on $(0, \alpha]$ and satisfy $v_{1}(0)=-c_{1} / D^{\prime}(0)$ and $v_{2}(0)=-c_{2} / D^{\prime}(0)$, then $v_{1}(\phi)>v_{2}(\phi) \forall \phi \in(0,1)$.

By using the phase portrait of (5) for $c=0$, the continuity of the vector field defined in (5) with respect to $c$, the vector field itself and the above analysis we can prove the following lemma: 


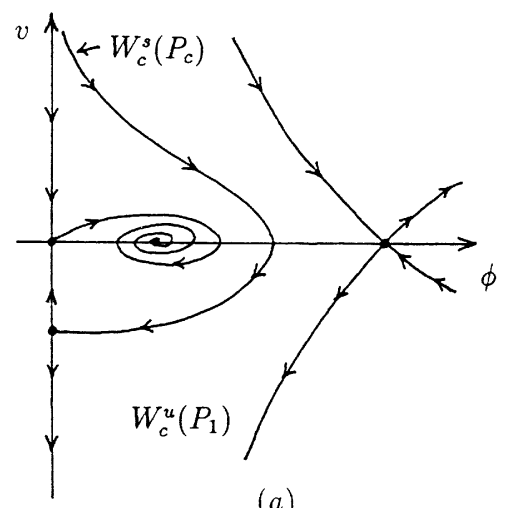

(a)

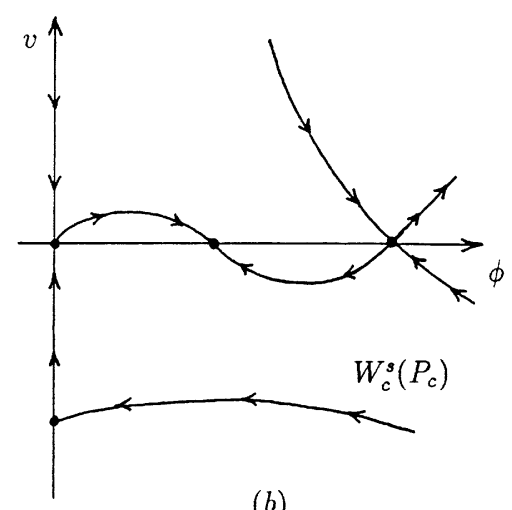

(b)

Fig. 6a, b. Phase portrait of (5) for extremum values of $c$. a Sufficiently small values of $c$ and b $c^{2} \geqq \max \left[4 D^{\prime}(\phi) g(\phi)\right]$

Lemma 5.2. For $c>0$, system (5) does not have a heteroclinic trajectory from:

1. $P_{0}$ to $P_{1}$ for $\mathscr{D}(1)>0$,

2. $P_{1}$ to $P_{0}$ for $\mathscr{D}(1) \leqq 0$.

In terms of the t.w.s. dynamics of (1), Lemma 5.2 means that for $c \geqq 0$ there are no t.w.s. satisfying: $\phi(-\infty)=0$ and $\phi(+\infty)=1$ for $\mathscr{D}(1)>0$ and 2 . $\phi(-\infty)=1$ and $\phi(+\infty)=0$ for $\mathscr{D}(1) \leqq 0$. Hence item 4 . in Theorem 1 is proved.

By noting that the function

$$
V(\phi, v)=\frac{1}{2}[D(\phi) v]^{2}+[\mathscr{D}(\phi)-\mathscr{D}(\alpha)]
$$

is a Lyapunov function for (5) for the equilibrium $P_{\alpha}$ and by considering the behaviour of the trajectories of (5) approaching the horizontal axis coming from $P_{0}$ and from $P_{1}$, we can prove the following lemma:

Lemma 5.3. 1. If $\mathscr{D}(1)>0$ then for each $c>0$, (5) has a heteroclinic trajectory from $P_{0}$ to $P_{\alpha}$ which: a) oscillates around $P_{\alpha}$ if $0<c<\sqrt{4 D(\alpha) g^{\prime}(\alpha)}$ or b) is monotone if $c \geqq \sqrt{4 D(\alpha) g^{\prime}(\alpha)}$.

2. If $\mathscr{D}(1) \leqq 0$, then for each $c>0$, (5) has a heteroclinic trajectory from $P_{1}$ to $P_{\alpha}$ which: a) oscillates around $P_{\alpha}$ if $0<c<\sqrt{4 D(\alpha) g^{\prime}(\alpha)}$ or b) is monotone if $c \geqq \sqrt{4 D(\alpha) g^{\prime}(\alpha)}$.

The above lemma contains information on the t.w.s. dynamics of (1) but, given that the bounds of $c$ will be refined in the next section, we do not state the result in those terms.

We now proceed to analyse more closely the dynamics of the manifolds $W_{c}^{u}\left(P_{1}\right)$ and $W_{c}^{s}\left(P_{c}\right)$ for the case $\mathscr{D}(1)>0$ as $c$ increases. 


\section{The existence and uniqueness of a sharp front}

We start by considering large values of $c$. The following proposition holds:

Proposition 6.1. For $c$ such that $c^{2} \geqq \max \left[4 D^{\prime}(\phi) g(\phi)\right]$ the manifold $W_{c}^{s}\left(P_{c}\right)$ leaves (in reverse time) the region $\mathscr{R} \equiv\{(\phi, v) \mid 0<\phi<1, v<0\}$ somewhere on $\{(\phi, v) \mid \phi=1, v<0\}$. Meanwhile for all $c \geqq 0, W_{c}^{u}\left(P_{1}\right)$ enters the region $\mathscr{R}$.

Proof. For the first part the reasoning is similar to that given in [20], Lemma 1. It uses the vector field defined by (5) and the vertical null-clines (10) shown in Fig. 5. The second part is immediate by using the local linear analysis around $P_{1}$ developed in Sect. 3.

Proposition 6.1 allows us to conclude that for the above values of $c$ the manifold $W_{c}^{u}\left(P_{1}\right)$ entering the region $\mathscr{R}$ has, in principle, two possible behaviours as $\tau \rightarrow \infty$ : either it tends to $P_{\alpha}$ or it tends to $P_{0}$. Now we clarify the actual behaviour of $W_{c}^{u}\left(P_{1}\right)$ and $W_{c}^{s}\left(P_{c}\right)$ for $c>0$.

To begin, note that for all $u \in[\alpha, 1]$ equation (1) is a non-degenerate Fisher-KPP equation and one can use the t.w.s. analysis carried out in [8] and [12], which reduces the analysis for equation $u_{t}=\left[D(u) u_{x}\right]_{x}+g(u)$ to that for $u_{t}=u_{x x}+D(u) g(u)$. Hence we can use the result for the ODE system of those equations, to prove the following lemma:

Lemma 6.1. For each $c \geqq \bar{c}$ with $\bar{c}$ such that

$$
\sqrt{4 D(\alpha) g^{\prime}(\alpha)} \leqq \bar{c} \leqq \sqrt{\sup \left[4 \frac{D(\phi) g(\phi)}{(\phi-\alpha)}\right]},
$$

where the sup is taken on $\phi \in(\alpha, 1)$, system (5) has two:

1. Damped heteroclinic trajectories for $\bar{c}=\sqrt{4 D(\alpha)^{\prime}}(\alpha)$ : one from $P_{1}$ to $P_{\alpha}$ and the other from $P_{0}$ to $P_{\alpha}$,

2. Monotone heteroclinic trajectories for $\bar{c}>\sqrt{4 D(\alpha) g^{\prime}(\alpha)}$ : one from $P_{1}$ to $P_{\alpha}$ and the other from $P_{0}$ to $P_{\alpha}$.

Now let us consider small values of $c$. We can prove the following proposition:

Proposition 6.2. Suppose that $\mathscr{D}(1)>0$. Then, for sufficiently small (positive) values of $c$ :

1. $W_{c}^{u}\left(P_{1}\right) \rightarrow(0,-\infty)$ as $\tau \rightarrow \infty$,

2. $W_{c}^{s}\left(P_{c}\right)$ leaves (in reverse time) the region $\mathscr{R}$ somewhere on $(\alpha, 1)$,

3. The trajectory leaving $P_{0}$ ends at $P_{\alpha}$.

Proof. Items 1 and 2 follow by using continuity of the solutions of (5) with respect to the speed $c$ and Lemma 4.1 (items 1 and 2, respectively). Item 3 follows by using Lemma 6.1 .

So far in this section we have analysed the behaviour of $W_{c}^{s}\left(P_{c}\right)$ and $W_{c}^{u}\left(P_{1}\right)$ for small and large values of $c$. Now we consider intermediate values of $c$. For this, we can use a couple of results proved previously (see [20]). 
Let $\left(\phi_{c}, v_{c}\right)$ be the point where the manifold $W_{c}^{s}\left(P_{c}\right)$ leaves (in reverse time) the region $\mathscr{R}$. We define

$$
c^{*}=\inf \left\{c \mid c>0, \phi_{c}=1, v_{c}(1)<0\right\},
$$

to prove the following lemma:

Lemma 6.2 (Existence and Uniqueness). For $\mathscr{D}(1)>0$, there exists a value, $c^{*}>0$, of $c$, such that the trajectory $W_{c^{*}}^{s}\left(P_{c^{*}}\right)$ is the unique trajectory connecting (in reverse time) the point $P_{c^{*}}$ to the point $P_{1}$.

Proof. This follows the same reasoning as that given in [20], so we omit the details.

By using continuity arguments on the solutions of (5) with respect to the parameter $c$ and all the results proved in the above sections, we can summarize the dynamics of the heteroclinic connections of (5) for $\mathscr{D}(1)>0$ and $c>0$ as follows:

Lemma 6.3. For $\mathscr{D}(1)>0$, (5) has:

1. No heteroclinic trajectory from $P_{0}$ to $P_{1}$ for $c>0$,

2. An oscillatory heteroclinic trajectory (oscillating around $P_{\alpha}$ ) connecting $P_{0}$ with $P_{\alpha}$ for each $c$ such that $0<c<c^{*}<\sqrt{4 D(\alpha) g^{\prime}(\alpha)}$,

3. Two heteroclinic trajectories for $c=c^{*}$ : one of oscillatory type (oscillating around $P_{\alpha}$ ) from $P_{0}$ to $P_{\alpha}$ and one of saddle-saddle type connecting $P_{1}$ with $P_{c^{*}}$,

4. Two oscillatory (oscillating around $P_{\alpha}$ ) heteroclinic trajectories for $c^{*}<c<\sqrt{4 D(\alpha) g^{\prime}(\alpha)}$ : one from $P_{0}$ to $P_{\alpha}$ and the other from $P_{1}$ to $P_{\alpha}$,

5. Two heteroclinic trajectories for $c \geqq \sqrt{4 D(\alpha) g^{\prime}(\alpha)}$ : one from $P_{0}$ to $P_{\alpha}$ and the other from $P_{1}$ to $P_{\alpha}$. Both are damped when equality occurs and they are monotone in the case of strict inequality.

If we interpret each of the heteroclinic trajectories, whose existence has already been proved above, as the corresponding different types of t.w.s. for equation (1), we conclude the proof of Theorem 1.

We illustrate the analytical results obtained in the above sections by considering an example:

Example 2. Here we consider equation (1) with $D(u)=\beta u+u^{2}$ and $g(u)=u(1-u)(u-\alpha)$, where $\beta>0$ and $\alpha \in(0,1)$.

The corresponding non-singular ODE system (5) takes the form

$$
\begin{aligned}
\dot{\phi} & =\left(\beta \phi+\phi^{2}\right) v \\
\dot{v} & =-c v-(\beta+2 \phi) v^{2}-\phi(1-\phi)(\phi-\alpha) .
\end{aligned}
$$

The equilibria for this system are: $P_{0}=(0,0), P_{1}=(1,0), P_{\alpha}=(\alpha, 0)$ and $P_{c}=\left(0,-c / D^{\prime}(0)\right)$. Figure 7 shows the phase portrait of (17) for different 

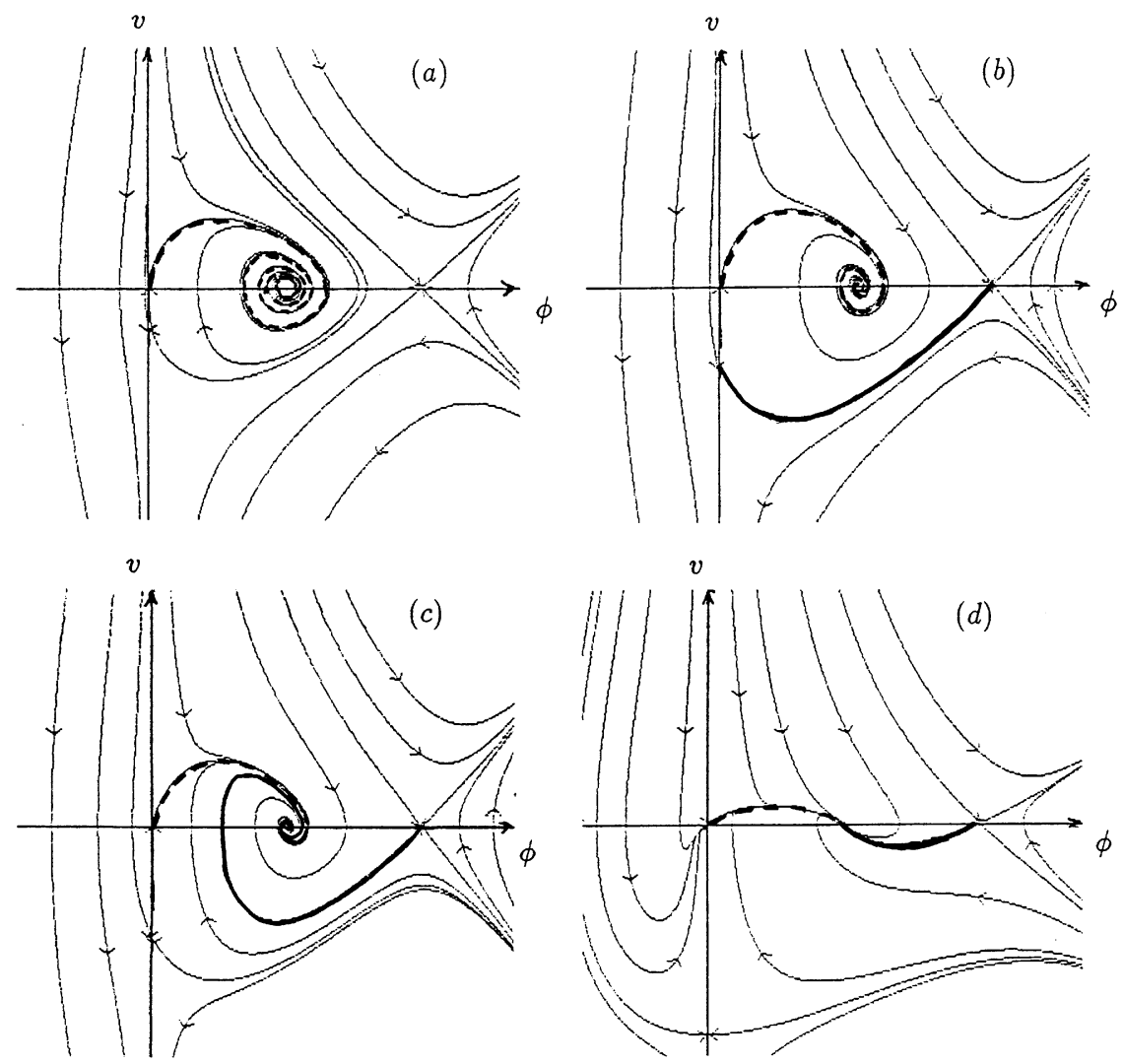

Fig. 7a-d. Phase portrait of system (17) for different values of $c$ : a $c=0.1$ : Here there exists only the $P_{0}$ to $P_{\alpha}$ connection (thick broken line). $\mathbf{b} c=0.201$ : This is an approximation to the critical value of $c$ for which there exists a saddle $\left(P_{1}\right)$-saddle $\left(P_{c}\right)$ heteroclinic trajectory (thick line). Also for this value of $c$ we have an oscillatory connection from $P_{0}$ to $P_{\alpha}$ (thick broken line). c $c=0.3$ : Here there are two oscillatory heteroclinic trajectories; from $P_{0}$ to $P_{\alpha}$ (thick broken line) and from $P_{1}$ to $P_{\alpha}$ (thick line). d $c=1.0$ : Here there are two monotonic heteroclinic connections, one from $P_{0}$ to $P_{\alpha}$ (thick broken line) and the other from $P_{1}$ to $P_{\alpha}$ (thick line)

values of $c$ with $\beta=2$ (the qualitative features of the phase portrait do not change with $\beta$ ). The dynamics shown in Fig. 7 agrees with the analytical results contained in this paper. Note that for $c \approx 0.201$ we have the saddlesaddle heteroclinic connection. Associated with this trajectory we have the sharp type solution for the corresponding equation (1) with $D$ and $g$ as above (see [20] for the definition of sharp type solution).

Numerical simulations of the partial differential equation strongly suggest that the sharp type solution is stable for a step function initial condition and the final speed attained by the wave agrees very closely with that predicted by the phase portrait analysis corresponding to the saddle-saddle heteroclinic trajectory. 


\section{Conclusions and discussion}

We conclude this paper addressing the points below:

1. The rich dynamics of system (5) comes mainly from the geometric properties of $g$. It is remarkable how this leads to a wider range of heteroclinic and homoclinic trajectories compared with those of the degenerate Fisher-KPP equation (see [20]). Consequently, equation (1) exhibits a greater richness of t.w.s. behaviour than that of the degenerate Fisher-KPP equation.

2. The degeneracy of equation (1) is responsible for the existence of the sharp type solution for that equation, while the uniqueness comes from the monotonicity of the solutions of equation (11) with respect to $c$.

3. The numerical simulations carried out on the full PDE suggest that for a step function initial condition, the sharp travelling wave solution of (1), for $D$ and $g$ given in Example 2, is stable. The rigorous proof of convergence to the different types of t.w.s. for the general equation (1), whose existence has been proved in this paper, is still an open problem.

Acknowledgements. FSG thanks the Dirección General de Asuntos del Personal Académico $(D G A P A)$ ) and the Facultad de Ciencias both departments of the Universidad Nacional Autónoma de México $(U N A M)$ for their support during this investigation. He also thanks the hospitality of the Centre for Mathematical Biology at Oxford where part of this work was developed. PKM would like to thank the Department of Mathematics, Williams College, Massachusetts for their support and hospitality.

\section{References}

1. Andronov, A. A., Leontovich, E. A., Gordon I. I. and Maier, A. G.: Theory of Dynamic Systems on a Plane (Israel Program for Scientific Translations, Jerusalem, 1973)

2. Aronson D. G. and Weinberger, H. F.: Nonlinear Diffusion in population, genetics and nerve pulse propagation, in Partial Differential Equations and Related Topics, Lecture Notes in Mathematics 446, ed. J.A. Goldstein (Springer Verlag 1975)

3. Aronson, D. G.: Density-Dependent Interaction-Diffusion Systems, in Dynamics and Modelling of Reactive Systems, eds. Steward, W. E., Ray W. H. and Conley C. C. (Academic Press, 1980) 161-176

4. Aronson, D. G.: The role of the diffusion in mathematical population biology: Skellam revisited, in Lecture Notes in Biomathematics 57, eds. V. Capasso, E. Grosso and S.L. Naveri-Fontana (Springer Verlag, 1985)

5. Arrowsmith, D. K. and Place, C. M.: An Introduction to Dynamical Systems (Cambridge University Press, 1990)

6. Carl, E. A.: The Population Control in Arctic Ground Squirrels, Ecology, Vol 52, No. 3, 395-413

7. Carr, J.: Applications of Centre Manifold Theory, (Springer Verlag 1981).

8. Engler, H.: Relations Between Travelling Wave Solutions of Quasilinear Parabolic Equations, Proc. Am. Math. Soc., 93, 297-302 (1985)

9. Gurtin, M. E. and MacCamy, R. C.: On the Diffusion of Biological Populations, Math. Biosci., 33, 35-49 (1977)

10. Fisher, R. A.: The Wave of Advance of Advantageous Genes, Ann. Eugenics, 7, 353-367 (1937)

11. Grindrod, P. and Sleeman, B. D.: Weak Travelling Fronts for Population Models with Density-Dependent Dispersion, Math. Meth. in the Appl. Sci., 9, 576-586 (1987) 
12. Hadeler, K. P.: Free Boundary Problems in Biology, in Free Boundary Problems: Theory and Applications Vol. II, Eds. A. Fasano and M. Primicerio, (Pitman Advanced Publishing Program, 1983)

13. Hosono, Y.: Travelling Wave Solutions for Some Density Dependent Diffusion Equations, Japan J. Appl. Math., 3, 163-196 (1986)

14. Kappos, E., Hutson, V. C. L. and Michaikow, K.: Conley Index Approach to the Existence of Travelling Wave Solutions for a Scalar Degenerate Reaction-Diffusion Equation (Manuscript 1992)

15. Kolmogoroff, A., Petrovsky, I and Piskounoff, N.: Étude de l'équation de la diffusion evec croissance de la quatité de matiere et son application a un probleme biologique, Moscow Univ. Bull. Math., 1, 1-25 (1937)

16. Murray, J. D.: Mathematical Biology, Biomathematics Texts 19 (Springer Verlag 1989)

17. Myers, J.H. and Krebs, Ch. J.: Population Cycles in Rodents, Sci. Am., 6, 38-46 (1974)

18. Rothe, F.: Asymptotic Behaviour of the Solutions of the Fisher Equation in Lecture Notes in Biomathematics 38, eds. W. Jager, H. Rost and P. Tautu, (Springer-Verlag, 1980)

19. Sánchez-Garduño, F.: Travelling Waves in One-Dimensional Degenerate Non-Linear Reaction-Diffusion Equations, D.Phil. thesis, University of Oxford (1993)

20. Sánchez-Garduño F. and Maini, P. K.: The Existence and Uniqueness of a Sharp Travelling wave Solution for a Generalized Fisher-KPP Equation, J. Math. Biol., 33, 163-192 (1994)

21. Sánchez-Garduño, F. and Maini, P. K.: Travelling Wave Phenomena in some Degenerate Reaction-Diffusion Equations, J. Diff. Eq., 117, 281-319 (1995) 